\title{
A Simple Genetic Algorithm for Calibration of Stochastic Rock Discontinuity Networks
}

\author{
R. Jimenez • R. Jurado-Piña
}

\begin{abstract}
We present a novel approach for calibration of stochastic discontinuity network parameters based on genetic algorithms (GAs). To validate the approach, examples of application of the method to cases with known parameters of the original Poisson discontinuity network are presented. Parameters of the model are encoded as chromosomes using a binary representation, and such chromosomes evolve as successive generations of a randomly generated initial population, subjected to GA operations of selection, crossover and mutation. Such backcalculated parameters are employed to make assessments about the inference capabilities of the model using different objective functions with different probabilities of crossover and mutation. Results show that the predictive capabilities of GAs significantly depend on the type of objective function considered; and they also show that the calibration capabilities of the genetic algorithm can be acceptable for practical engineering applications, since in most cases they can be expected to provide parameter estimates with relatively small errors for those parameters of the network (such as intensity and mean size of discontinuities) that have the strongest influence on many engineering applications.
\end{abstract}

\section{Introduction}

It is well known that discontinuities are the single individual factor with the strongest influence on the deformability, strength and permeability of rock masses (Hudson and Harrison 1997; Goodman 1976). However, despite recent contributions for three-dimensional characterization of fracture networks at block scale (see e.g. Dowd et al. 2009), deterministic characterization of individual discontinuities in a rock mass at engineering scale is usually unachievable in real applications.

Such difficulties to characterize individual discontinuities deterministically led to the development of stochastic discontinuity networks. Within this framework, the stochastic nature of discontinuities in a rock mass is considered in a statistical sense, that is, by means of statistical distributions (often constrained by a suitable geological model) that characterize their properties such as, for instance, location, size and termination mode (see e.g. Meyer and Einstein 2002; Lee et al. 1990; Dershowitz and Einstein 1988; Cravero et al. 2006).

To be able to use stochastic discontinuity networks in real applications, however, it is necessary to calibrate the parameters of the stochastic discontinuity network. In this regard, significant efforts have been made to characterize several aspects of discontinuities or discontinuity sets in the network, such as their intensity (Dershowitz and Herda 1992; Zhang and Einstein 2000), the distribution of their sizes (see e.g. Priest 2004; Lyman 2003b; Zhang et al. 2002; Villaescusa and Brown 1992; Kulatilake and $\mathrm{Wu}$ 1986; Tonon and Chen 2007; Jimenez-Rodriguez and Sitar 2006a) or their orientation (see e.g. Jimenez-Rodriguez and Sitar 2006b; Jimenez 2008; Tokhmechi et al. 2011). In addition, calibrated networks have been employed to characterize rock masses for different engineering 
applications, such as the design of underground nuclear repositories (Munier 2004; Kulatilake et al. 1993, 2003), analyses of groundwater flow (Meyer and Einstein 2002) and analysis of the formation of removable and unstable blocks in underground excavations (Kuszmaul 1999; Dershowitz and Carvalho 1996; Song et al. 2001; Starzec and Andersson 2002) and in slopes (Jimenez-Rodriguez and Sitar 2008; Park et al. 2005; Park and West 2001).

La Pointe et al. (1993) proposed a methodology based on forward modelling and simulated sampling to estimate the size of fractures in discontinuity networks. To that end, they simulate realizations of the stochastic network using different sets of parameters, and they compare statistics of the simulated trace maps with statistics of the original trace map. To perform such comparison, they build on the key idea that the ratio between the number of fractures partially intersecting one sampling surface to all fractures intersecting such surface is controlled by the size distribution of the discontinuity sets only. This assumption allows them to assess the 'quality' of a specific set of parameter values by comparing their (simulated) intersection ratios with the (observed) ratios of the original trace map. Similar ideas based on counting the number of traces within a sampling domain have also been employed to estimate the distribution of trace lengths in rock outcrops (see e.g. Pahl 1981; Mauldon 1998; Zhang and Einstein 1998; Lyman 2003a).

Genetic algorithms (GAs) are a special type of algorithm based on the ideas of evolutionary biology and natural selection (Mitchell 1996; Goldberg 1989). To solve a specific optimization problem, GAs consider an initial population of individuals as possible solutions, in which the parameters of each possible solution are suitably encoded by means of 'chromosomes'. [Note that we limit our discussion of GAs to optimization problems, although they have also been employed to solve other types of 'nonoptimization' problems; see Mitchell (1996) for additional examples.] The set of chromosomes that form each possible solution is the 'genotype' of such solution; accordingly, once decoded, the genotype of each individual represents one possible solution to the optimization problem. (Once decoded, the parameters that form such solution are called the 'phenotype'.)

One reason for the success of GAs is that the genotype of each individual is transmitted to future generations through a process of selection, crossover and mutation in such a way that 'fittest' individuals (hence the best available solutions to the problem up to that moment) have more probability of transmitting their genotype (in a probably slightly modified form) to future generations. As a consequence, each generation is expected to improve (in an average sense) its quality as a solution, hence increasing the probability of converging to an acceptable solution of the optimization problem. Note, however, that GAs are not guaranteed to provide a global optimum solution because of their inherent heuristic character, even though they have been shown to be excellent tools to solve complex optimization problems (Levasseur et al. 2008).

In the context of geotechnics, GAs have been employed (among others) to solve such different problems as parameter identification of soil and rock models (see e.g. Guan et al. 2009; Levasseur et al. 2008, 2010), identification of critical slip surfaces in slope stability (see e.g. Zolfaghari et al. 2005; Xue and Gavin 2007; Fahd and Jimenez 2008) and the reliability of finite elements (FE) designs (Cui and Sheng 2005). Similarly, in rock mechanics, GAs have been employed to identify the discontinuity frequency in fractured rock masses (Simpson and Priest 1993), for discontinuity clustering and estimation of discontinuity orientation (Kemeny and Post 2003; Cai et al. 2005) and to estimate the size and shape of rectangular fractures with constant size and aspect ratio (Decker and Mauldon 2006).

In this paper, we present an extension to the work of $\mathrm{La}$ Pointe et al. (1993) for calibration of rock discontinuity networks, and we propose a novel approach for inference of the network parameters based on the use of genetic algorithms. Similarly, and as a proof of concept, we validate the approach using discontinuity networks generated with the Poisson disk model and a simple genetic algorithm in which only reduced efforts for optimization have been employed. Our results show that GAs are able to successfully back-calculate the original parameters of the Poisson model used for generation of the reference stochastic discontinuity network, and that such estimation can be conducted with a degree of accuracy that is acceptable for many engineering applications.

\section{Generation of Discontinuity Traces}

We employ the Poisson disk model to generate discontinuities in a three-dimensional (3D) space. The Poisson disk model is a simple model commonly used to generate discontinuity networks in rock mechanics (see e.g. Baecher et al. 1977; Dershowitz and Einstein 1988); it has been found to generate discontinuities that are often similar to discontinuity patterns in nature, and it has been recognized that, in many cases, discontinuity networks are best characterized by Poisson models (La Pointe 1993; Bonnet et al. 2001).

Discontinuity centres generated with the Poisson disk model are assumed to be uniformly located within a 'generation domain' employed for discontinuity generation in $3 \mathrm{D}$, and discontinuity traces are obtained as the intersection of such discontinuities with a 'reference outcrop'. (Note that, therefore, the distribution of discontinuity trace lengths is not an input of the analysis, as it depends on the 
Fig. 1 Example of traces formed by the intersection of simulated 3D discontinuities with the 'reference outcrop'. The disk on the left represents a discontinuity that does not intersect the excavated slope. The disks on the right represent discontinuities that intersect the reference outcrop producing discontinuity traces with different censoring conditions

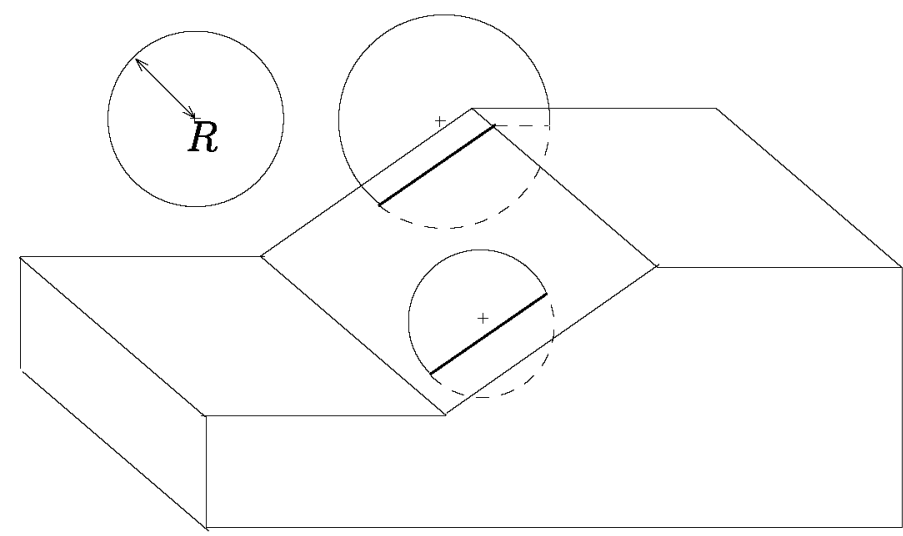

shape and size distribution of the 3D discontinuities considered.)

Figure 1 shows an example of (two-dimensional, 2D) discontinuity traces formed by the intersection of (3D) simulated discontinuities with a (rectangular) reference outcrop. Discontinuities are further assumed to be circular, with their radii following a lognormal distribution with mean $\mu_{\mathrm{R}}$ and standard deviation $\sigma_{\mathrm{R}}$. The amount of discontinuities is controlled by the volumetric intensity $P_{32}$ $\left[\mathrm{m}^{2} / \mathrm{m}^{3}\right.$ ] of generated discontinuities [defined as the ratio between area of discontinuities and rock volume; see Dershowitz and Herda (1992)], with discontinuities being generated until a threshold value of $P_{32}$ is achieved.

To calibrate the Poisson disk model described above, we need information about the orientation, the intensity and the size of discontinuities. The orientation of discontinuities, however, can be characterized without the need for a GA using well-established methods for identification of discontinuity sets and for characterization of their orientation (see e.g. Jimenez-Rodriguez and Sitar 2006b; Jimenez 2008, and references therein); for this reason, in our analyses below, discontinuities will be assumed to have a known (and constant) orientation. (For simplicity, discontinuities are further assumed to be perpendicular to the reference outcrop and parallel to one of the sides of the reference outcrop; for this reason, they are represented as circles in the projection shown in Fig. 1.)

Depending on whether the discontinuity intersects the reference outcrop, and (if it does) on the censoring conditions of the resulting discontinuity traces in relation to the reference outcrop, we can identify four types of discontinuities: (i) discontinuities that do not intersect the reference outcrop ('no intersection' or $C=-1$ conditions), (ii) discontinuities that intersect the outcrop producing traces with both ends observed ('no censoring', or $C=0$ conditions), (iii) discontinuities that intersect the outcrop producing traces with one end observed and one end censored ('censoring on one side', or $C=1$ conditions), and (iv) discontinuities that intersect the outcrop producing traces

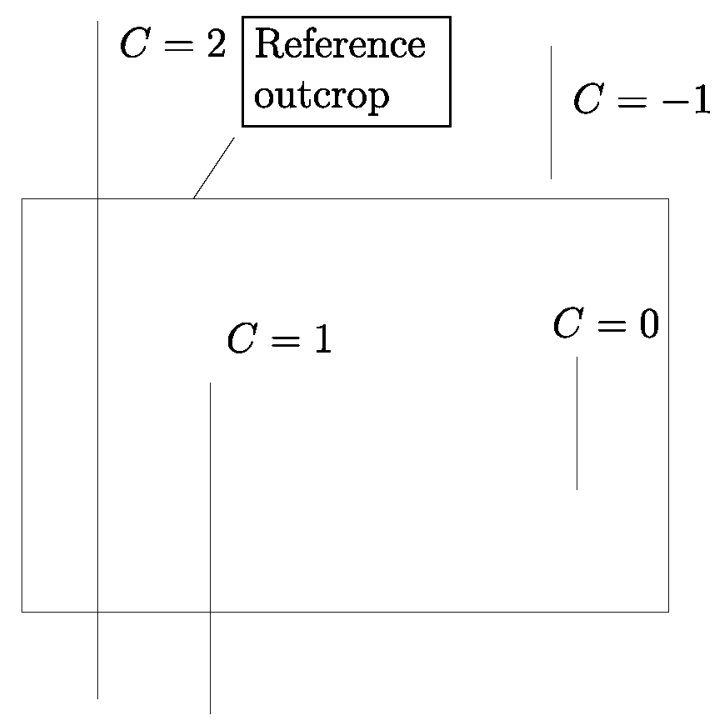

Fig. 2 Illustration of several censoring conditions for a simplified case

with both ends censored ('censoring on both sides', or $C=2$ conditions). Figure 2 shows an example of such censoring conditions for our simplified case with vertical discontinuity traces on a rectangular outcrop.

\section{Description of the Genetic Algorithm}

Genetic algorithms are evolutionary algorithms in which individuals of a population represent (using an adequate encoding by means of chromosomes) possible solutions to a particular problem. The genotypes of individuals are then modified using operations that mimic natural selection; the objective is to increase the 'fitness' of individuals in the population as solutions to a particular problem as the number of generations increases, thereby obtaining an acceptable (although probably not 'the best') solution to the problem. 
Fig. 3 Parameters represented by the chromosomes of individuals and illustration of the decoding operation of such chromosomes into real parameter values

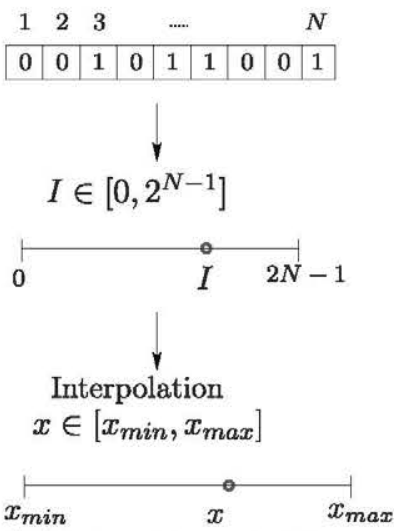

(a) Parameters represented by chromosomes of binary strings (b) Decoding of a binary string into a real number by interpolation within a range
Next, we present a description of the simple genetic algorithm employed in this work, which is heavily based on the work of Goldberg (1989). It has to be noted that we do not aim to optimize the different operations described, as the main aim of this paper is to present, as a proof of concept, that GAs are a viable tool for calibration of stochastic discontinuity networks. However, as we will show, acceptable results (from an engineering viewpoint) are obtained even with this relatively simple GA.

\subsection{Codification of Solutions}

The selection of an adequate encoding system is a crucial step for successful application of genetic algorithms in real applications (Mitchell 1996; Goldberg 1989). Based on the above, and as shown in Fig. 3a, our genetic algorithm will only need to consider individuals with a genotype that includes chromosomes to represent the intensity of discontinuities (as measured by $P_{32}$ ) and the parameters of the distribution employed to characterize discontinuity sizes (such as, for instance, its mean $\mu_{\mathrm{R}}$ and standard deviation $\left.\sigma_{\mathrm{R}}\right)$. Such parameters are real numbers, which indicates that we need to encode real numbers in our genetic algorithm. Goldberg (1989) discusses encoding alternatives for GAs, concluding that use of binary encoding is a practical solution in many cases. Using binary encoding, a chromosome representing a specific parameter value is encoded using a string of $0 \mathrm{~s}$ and $1 \mathrm{~s}$; such string can then be easily decoded to an integer $I$ in the interval $\left[0,2^{N}-1\right]$, where $N$ is the length of the binary string representing the chromosome. Then, as shown in Fig. 3b, we can use $I$ to interpolate within a given interval where the solution for real parameter $x$ is expected; i.e., $x \in\left[x_{\min }, x_{\max }\right]$, where $x_{\min }$ and $x_{\max }$ are values that represent the user-specified search range. (The reader will note that we are in fact discretizing the solution search space; however, note also that the distance between two consecutive parameter values that can be obtained with this decoding operation is $\left(x_{\max }-x_{\min }\right) /\left(2^{N}-1\right)$, which is perfectly acceptable in real applications for large $N$ values, such as the $N=20$ employed in this work.)

\subsection{Objective Function}

The objective function quantifies the 'quality' (or fitness) of individuals as a solution to the optimization problem. To define such fitness, we need to work with the information that is available in each particular problem; this means that, in this case, we need to employ data available from discontinuity trace maps observed in rock outcrops. To that end, and working in a similar problem of estimation of discontinuity sizes, La Pointe et al. (1993) employed the ratio of discontinuities partially intersecting the sampling domain to all discontinuities intersecting such domain to evaluate the quality of a proposed size distribution as a solution to the inference problem.

Building on this idea, we choose to evaluate the similarity between trace maps by comparing the total number of traces between the observed and simulated trace maps, and also by comparing the number of discontinuity traces with $C=0$ (no censoring), $C=1$ (censoring on one side) and $C=2$ (censoring on both sides).

Furthermore, we allow some flexibility in the fitness function so that it can incorporate the differences between the observed and simulated mean length of discontinuity traces, and also between the observed and simulated standard deviation of discontinuity trace lengths. In particular, we define a general fitness function given by 


$$
\begin{aligned}
f= & w_{0}+\left(1.0+w_{1} \delta_{N_{\text {total }}}+w_{2} \delta_{N 0}+w_{3} \delta_{N 1}\right. \\
& \left.+w_{4} \delta_{N 2}+w_{5} \delta_{\mu_{\mathrm{L}}}+w_{6} \delta_{\sigma_{\mathrm{L}}}\right)^{-1},
\end{aligned}
$$

where $\delta_{N}=\frac{N^{\text {ref }}-N^{\mathrm{GA}}}{N^{\text {ref }}}$ is the relative error between the observed (reference) number of total or censored discontinuity traces and the number of such traces simulated with the GA ( $N_{\text {total }}$ indicates the total number of traces; and $N_{i}$, with $i=\{0,1,2\}$, indicates the number of traces with $C=i$ censoring conditions); $\delta_{\mu_{\mathrm{L}}}=\frac{\mu_{\mathrm{L}}^{\mathrm{ref}}-\mu_{\mathrm{L}}^{\mathrm{GA}}}{\mu_{\mathrm{L}}^{\text {ref }}}$ is the relative error between the observed (reference) and simulated (GA) mean length of discontinuity traces on the outcrop; $\delta_{\sigma_{\mathrm{L}}}=$ $\frac{\sigma_{\mathrm{L}}^{\text {ref }}-\sigma_{\mathrm{L}}^{\mathrm{GA}}}{\sigma_{\mathrm{L}}^{\text {ref }}}$ is the relative error in the standard deviation of observed and simulated discontinuity trace lengths; and $w_{i}$, with $i=\{0, \ldots, 6\}$, are weighting factors that allow us to change the relative importance of the different terms involved in the definition of the fitness function.

Although we will show that some simple fitness functions based on Eq. 1 produce adequate results while maintaining the simplicity of the algorithm, the reader should note that we do not postulate that Eq. 1 is the best possible fitness function and, for instance, we employ a linear 'fitness scaling' to improve convergence (Goldberg 1989). The motivation behind such correction is to avoid 'very good' individuals that could happen at initial generations from being selected too often (hence leading to premature 'specialization') and, at the same time, to promote the reproduction of the best individuals at later stages, when the average fitness is similar to the fitness of the best individuals [even though the 'diversity' of the population may still be significant; see Goldberg (1989)]. The linear fitness scaling is given by

$f^{\prime}=a f+b$,

where $f$ is the 'original' fitness computed with Eq. 1, and $f$ is the scaled fitness. Coefficients $a$ and $b$ can be selected in different ways; following Goldberg (1989), we impose two conditions so that (i) the original and scaled fitness have identical averages $\left(f_{\text {avg }}=f_{\text {avg }}^{\prime}\right.$ ), and (ii) that the maximum scaled fitness is a specified constant times the average $\left(f_{\max }^{\prime}=C_{\text {mul }} f_{\text {avg }}\right)$. Goldberg (1989) indicates that $C_{\text {mult }}$ values from 1.2 to 2.0 typically work well for 'small' populations of around 50-100, and we used $C_{\text {mult }}=1.7$ for our population of 120 , except when negative fitness values would have been produced. [In that case, $C_{\text {mult }}$ is selected so as to produce a minimum scaled fitness of zero; for additional discussion and code snippets, see Goldberg (1989).]

\subsection{Reproduction, Crossover and Mutation}

Reproduction, crossover and mutation are the fundamental operators that dictate the evolution of genotypes in a genetic algorithm. In particular, reproduction defines how 'parent' individuals are selected so that they can produce a new generation of 'descendant' individuals; crossover indicates how the genotypes of parent individuals are combined (or not) to produce the genotypes of descendants; and, finally, mutation produces small random variations of the genotype of some individuals.

The probability that a given individual is selected for reproduction increases as its fitness increases (or, in other words, as its quality as a solution increases). To that end, following the work of Goldberg (1989), we employ a roulette-wheel selection scheme (Fig. 4), in which the probability, $p_{i}$, of selection for reproduction of individual $i$, is given by its (scaled) fitness, $f_{i}^{\prime}$ (see Eq. 2), divided by the sum of the (scaled) fitness of all individuals in that generation; i.e.,

$p_{i}=\frac{f_{i}^{\prime}}{\sum_{j=1}^{N} f_{j}^{\prime}}$.

To select a specific individual for reproduction, we start by generating a random number $u$ from a uniform distribution $U[0,1]$, and we select for reproduction the individual in position $M$, where $M$ is the smallest integer such that the following equation holds:

$\sum_{i=1}^{M} p_{i}>u$

Crossover is also implemented following a simple operation proposed by Goldberg (1989), in which we take advantage of the encoding system employed (Fig. 5).

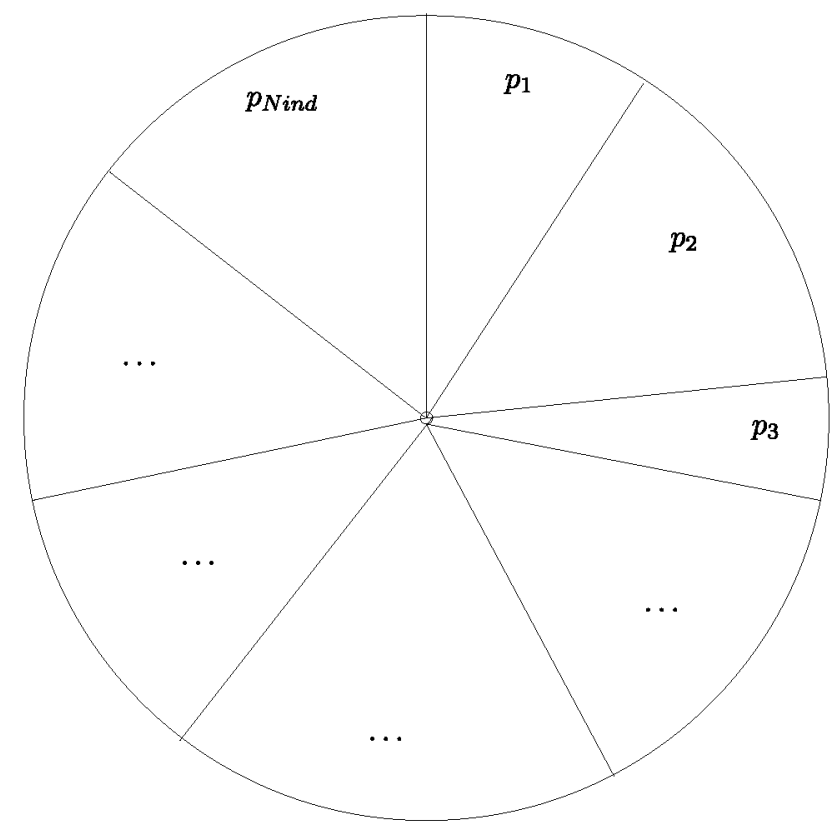

Fig. 4 Illustration of the roulette-wheel selection scheme for reproduction operation 
Fig. 5 Schematic

representation of the crossover operation (based on Goldberg 1989)
Fig. 6 Schematic representation of the mutation operation (based on Goldberg 1989)

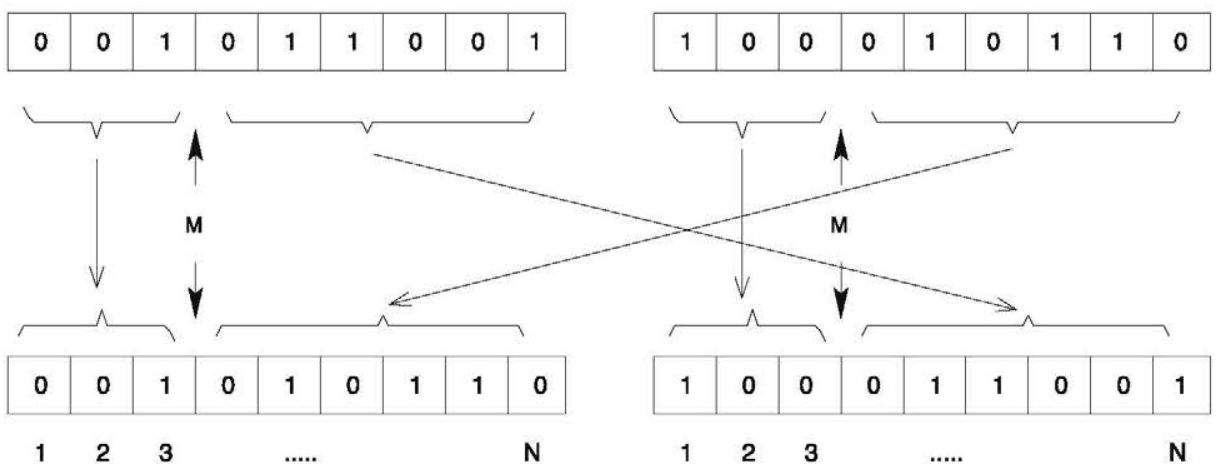

(a) If $u<P_{\text {crossover }} \Rightarrow$ Create offsprings with combined genetic information

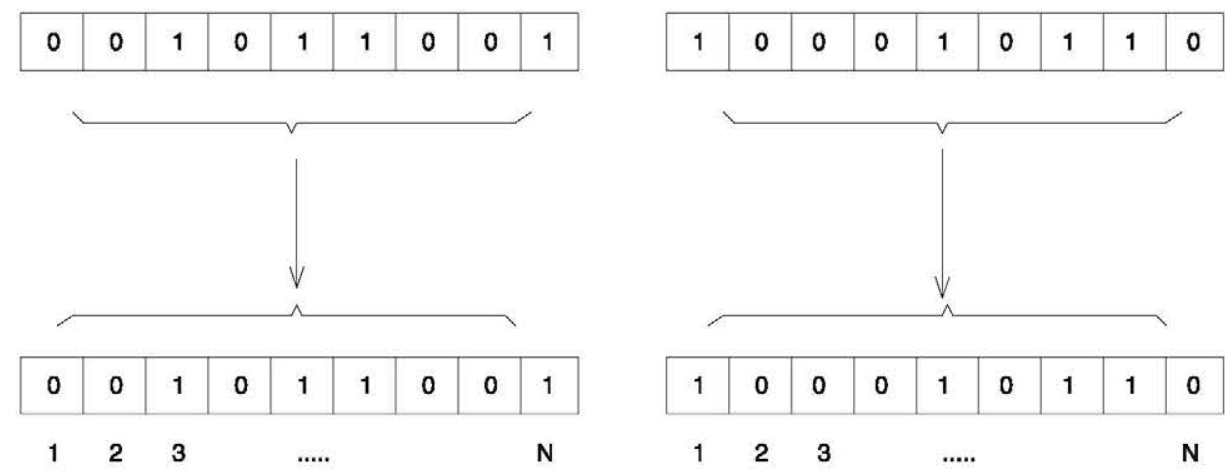

(b) If $u \geq P_{\text {crossover }} \Rightarrow$ Create offsprings with unchanged genetic information

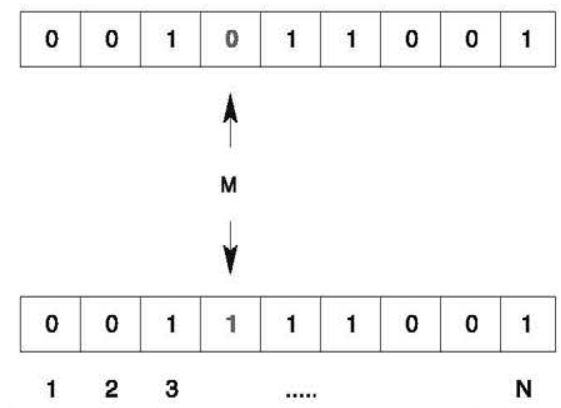

(a) If $u<P_{\text {mutation }} \Rightarrow$ Mutate chromosome

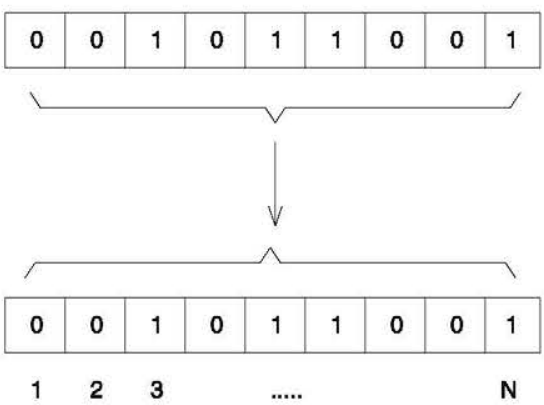

(b) If $u \geq P_{\text {mutation }} \Rightarrow$ Keep chromosome unchanged
Given two parent individuals, we first use a binomial distribution with parameter $P_{\text {crossover }}$ to decide whether crossover occurs. If the output is positive (which will happen, with probability $P_{\text {crossover }}$, when a random value $u$ generated from a uniform distribution $U[0,1]$ is such that $u<P_{\text {crossover }}$ ), then we combine the chromosomes of both parents to produce two offsprings with mixed genotypes (Fig. 5a); otherwise, we just copy the genetic content from parents to offsprings (Fig. 5b). To combine chromosomes, we first generate a random integer (which we call $M$ ) uniformly distributed in the interval $[1, N-1]$; once position $M$ has been determined, the crossover operation combines bit strings as indicated in Fig. 5a; that is, the first descendant's chromosome is produced by concatenation of the initial $M$ bits (zeros or ones) of the first parent's chromosome and the last $N-M$ bits of the second parent's chromosome; similarly, the second descendant's chromosome is produced by concatenating the initial $M$ bits of the second parent's chromosome and the last $N-M$ bits of the first parent's chromosome. 


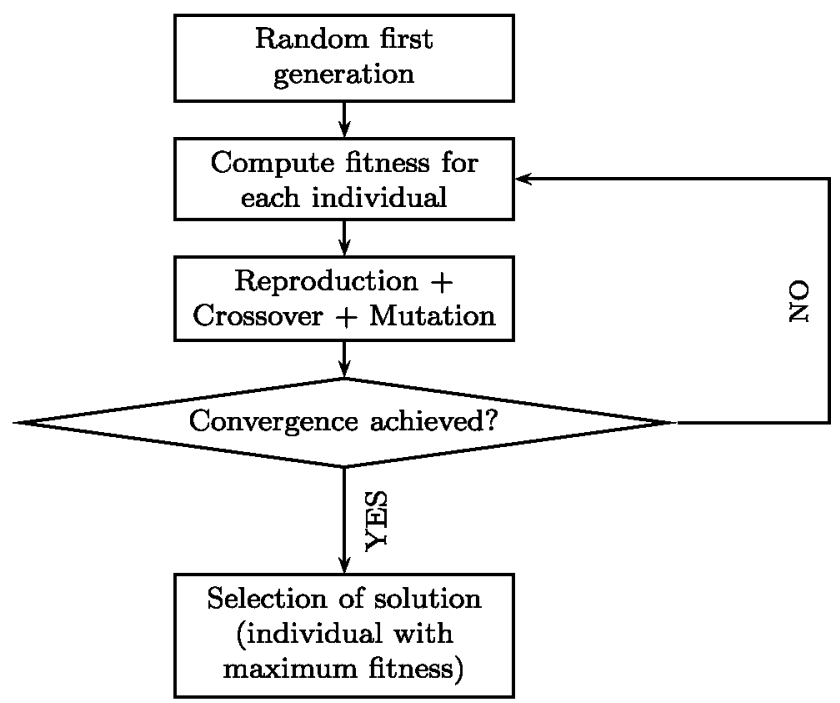

Fig. 7 Flow diagram of the simple genetic algorithm employed in this work

Finally, mutation of a given chromosome in a given generation will occur with probability $P_{\text {mutation }}$. To decide whether mutation occurs, we use a binomial distribution with parameter $P_{\text {mutation. If }}$. the output is positive and mutation occurs (which will happen with probability $P_{\text {mutation }}$, when a random value $u$ generated from a uniform distribution $U[0,1]$ is such that $u<P_{\text {mutation }}$ ), a random integer $M$ is generated with uniform probability in the interval $[1, N] . M$ indicates the location of the bit in the chromosome that will be mutated or 'flipped'; that is, as illustrated in Fig. 6a, if the $M$-th bit is 0 , it mutates to 1 ; and if it is 1 , it mutates to 0 . If, on the other hand, the outcome is negative (i.e., $u \geq P_{\text {mutation }}$ ), then nothing happens and the chromosome is kept unchanged (Fig. 6b).

\subsection{Representation of a Simple Genetic Algorithm}

Figure 7 shows a flow diagram with the structure of the simple genetic algorithm employed in this work. The process starts with the generation of $N_{\text {ind }}$ individuals with random genotypes. (That is, bits of chromosomes that represent each property of the stochastic discontinuity network are randomly filled with $0 \mathrm{~s}$ and $1 \mathrm{~s}$.) Next, we use an iterative process in which, for each generation, we compute its (scaled) fitness and we employ the reproduction, crossover and mutation operations to obtain new generations of individuals until a convergence criteria is achieved. (We work with a specified number of generations, but other stopping criteria based, for instance, on convergence of estimated parameter values or on convergence of the fitness function could have been employed as well.)

\section{Application Examples}

We tested the applicability of genetic algorithms for calibration of stochastic discontinuity networks using several example cases with the Poisson disk model. In addition, and to illustrate the influence of the objective (fitness) function and of the probabilities of crossover and mutation on the performance of the genetic algorithm, we performed several sets of analyses that are summarized in Table 1.

As indicated before, it is assumed that the orientation of discontinuity sets is known, which means that we only need to characterize the three remaining parameters of the network: intensity, and mean and standard deviation of the discontinuity size distribution. For each analysis (or GA) type listed in Table 1, several test cases with different reference (generation) parameter values were considered. [A lognormal distribution of discontinuity radii was considered in all cases for both generation and GA inference; for field data, in which case the type of distribution is generally not known, the selection of the 'best' distribution type is a complex task beyond the scope of this paper, although flexible distributions that incorporate little a priori information could be a promising approach to tackle this problem; see Jimenez-Rodriguez and Sitar (2006a) for an example of their application to the inference of trace length distributions.] The parameter values inferred (estimated) with each GA, as well as their corresponding relative errors, are listed in Table 2 . (In all cases, the GAs were defined to have $N_{\text {ind }}=120$ individuals and $N_{\text {gen }}=1,000$ generations.)

Table 1 Summary of GAs tested in this work

\begin{tabular}{|c|c|c|c|c|c|c|c|c|c|}
\hline Analysis & $w_{0}$ & $w_{1}$ & $w_{2}$ & $w_{3}$ & $w_{4}$ & $w_{5}$ & $w_{6}$ & $P_{\text {crossover }}$ & $P_{\text {mutation }}$ \\
\hline 1 & 1.0 & 1.5 & 1.0 & 1.0 & 1.0 & 0.0 & 0.0 & 0.85 & 0.06 \\
\hline 2 & 1.0 & 1.5 & $N_{0} / N_{\text {total }}$ & $N_{1} / N_{\text {total }}$ & $N_{2} / N_{\text {total }}$ & 0.0 & 0.0 & 0.85 & 0.06 \\
\hline 3 & 1.0 & 1.5 & 1.0 & 1.0 & 1.0 & 0.0 & 0.0 & 0.80 & 0.03 \\
\hline 4 & 1.0 & 1.5 & 1.0 & 1.0 & 1.0 & 0.0 & 0.0 & 0.95 & 0.03 \\
\hline 5 & 0.0 & 1.5 & $0.5+0.5 N_{0} / N_{\text {total }}$ & $0.5+0.5 N_{1} / N_{\text {total }}$ & $0.5+0.5 N_{2} / N_{\text {total }}$ & 0.0 & 0.0 & 0.85 & 0.06 \\
\hline 6 & 0.0 & 2.0 & $0.5+0.5 N_{0} / N_{\text {total }}$ & $0.5+0.5 N_{1} / N_{\text {total }}$ & $0.5+0.5 N_{2} / N_{\text {total }}$ & 1.0 & 1.0 & 0.85 & 0.06 \\
\hline 7 & 0.0 & 1.5 & $0.5+0.5 N_{0} / N_{\text {total }}$ & $0.5+0.5 N_{1} / N_{\text {total }}$ & $0.5+0.5 N_{2} / N_{\text {total }}$ & 0.0 & 0.0 & 0.95 & 0.03 \\
\hline
\end{tabular}




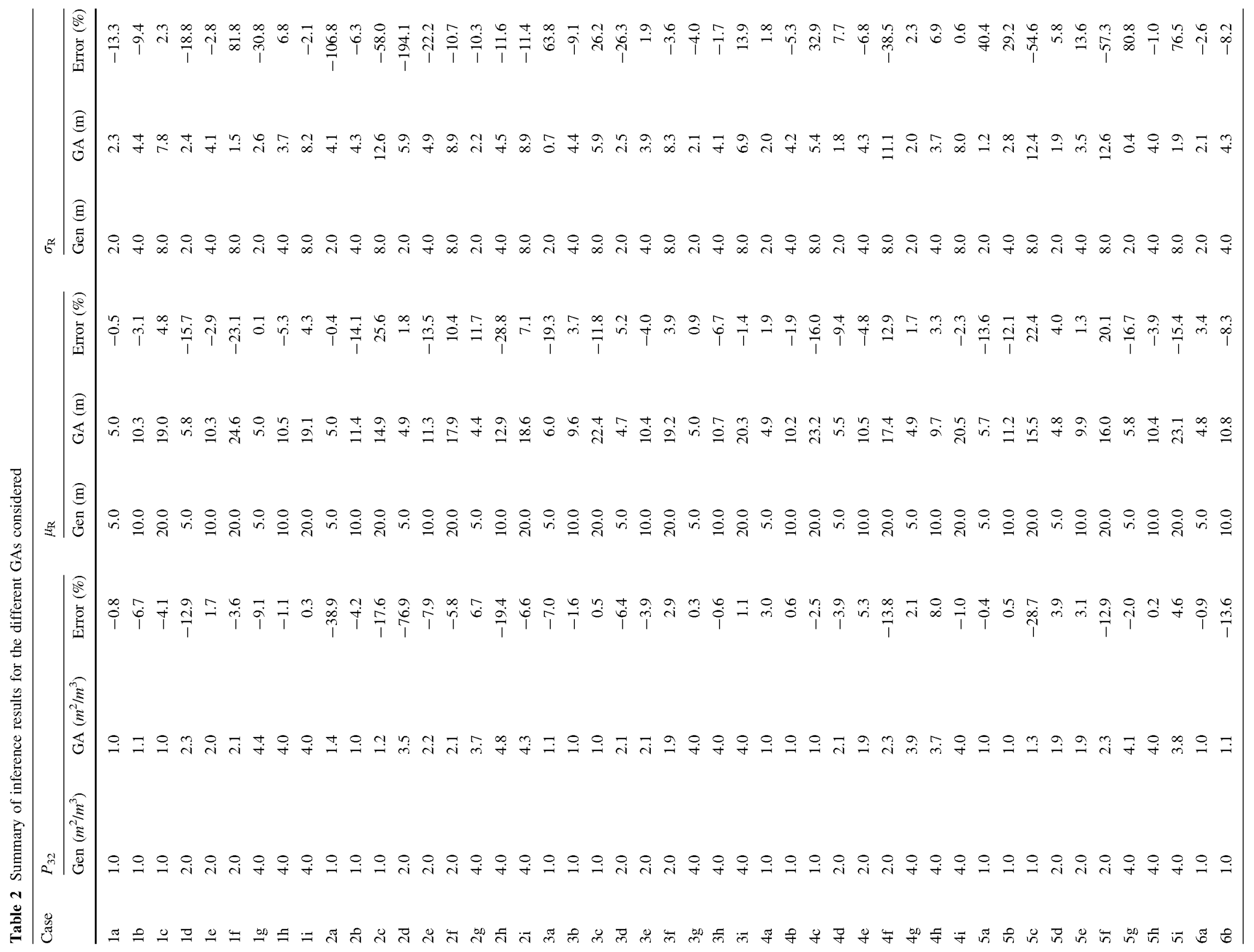


Table 2 continued

\begin{tabular}{|c|c|c|c|c|c|c|c|c|c|}
\hline \multirow[t]{2}{*}{ Case } & \multicolumn{3}{|l|}{$P_{32}$} & \multicolumn{3}{|l|}{$\mu_{\mathrm{R}}$} & \multicolumn{3}{|l|}{$\sigma_{\mathrm{R}}$} \\
\hline & $\operatorname{Gen}\left(m^{2} / m^{3}\right)$ & GA $\left(m^{2} / m^{3}\right)$ & Error $(\%)$ & Gen (m) & GA (m) & Error (\%) & Gen (m) & GA (m) & Error $(\%)$ \\
\hline $6 \mathrm{c}$ & 1.0 & 1.3 & -31.9 & 20.0 & 19.7 & 1.4 & 8.0 & 14.8 & -84.7 \\
\hline $6 d$ & 2.0 & 2.0 & 2.0 & 5.0 & 5.1 & -2.0 & 2.0 & 1.8 & 7.6 \\
\hline $6 e$ & 2.0 & 2.1 & -5.8 & 10.0 & 10.9 & -9.4 & 4.0 & 3.7 & 6.4 \\
\hline $6 f$ & 2.0 & 2.1 & -4.4 & 20.0 & 22.7 & -13.7 & 8.0 & 5.5 & 30.7 \\
\hline $6 \mathrm{~g}$ & 4.0 & 4.0 & 0.9 & 5.0 & 4.9 & 2.0 & 2.0 & 2.1 & -3.6 \\
\hline $6 \mathrm{~h}$ & 4.0 & 4.0 & 0.2 & 10.0 & 10.5 & -5.5 & 4.0 & 3.6 & 10.2 \\
\hline $6 \mathrm{i}$ & 4.0 & 3.8 & 5.0 & 20.0 & 22.9 & -14.4 & 8.0 & 4.1 & 48.6 \\
\hline $7 \mathrm{a}$ & 1.0 & 1.0 & -3.0 & 5.0 & 4.6 & 7.6 & 2.0 & 2.2 & -7.9 \\
\hline $7 \mathrm{~b}$ & 1.0 & 1.0 & 1.1 & 10.0 & 10.4 & -3.7 & 4.0 & 3.8 & 4.3 \\
\hline $7 \mathrm{c}$ & 1.0 & 1.0 & -1.8 & 20.0 & 23.1 & -15.7 & 8.0 & 5.3 & 34.1 \\
\hline $7 \mathrm{~d}$ & 2.0 & 1.9 & 3.5 & 5.0 & 4.5 & 9.2 & 2.0 & 2.2 & -7.7 \\
\hline $7 \mathrm{e}$ & 2.0 & 2.0 & 1.5 & 10.0 & 10.5 & -5.1 & 4.0 & 4.3 & -7.6 \\
\hline $7 \mathrm{f}$ & 2.0 & 1.9 & 2.6 & 20.0 & 22.0 & -10.0 & 8.0 & 4.2 & 47.6 \\
\hline $7 \mathrm{~g}$ & 4.0 & 3.9 & 1.5 & 5.0 & 5.8 & -16.2 & 2.0 & 0.4 & 77.9 \\
\hline $7 \mathrm{~h}$ & 4.0 & 4.1 & -2.9 & 10.0 & 9.9 & 0.6 & 4.0 & 4.1 & -3.4 \\
\hline $7 \mathrm{i}$ & 4.0 & 4.0 & -0.4 & 20.0 & 22.5 & -12.3 & 8.0 & 6.5 & 18.2 \\
\hline
\end{tabular}

It can be observed that the performance (i.e. the inference capabilities) of the GA varies widely, and also that the GAs corresponding to analyses 3 and 4 seem to produce, on average, the best inference results with the smallest estimation errors. In other words, results suggest that genetic algorithms with a simple fitness function (defined in terms of the unweighted number of total and of censored traces only, without the use of trace length information) and with a relatively low probability of mutation $\left(P_{\text {muta- }}\right.$ tion $=0.03$ ), seem to provide the best inference results for a relatively wide interval of crossover probabilities (with $P_{\text {crossover }}$ in the range between 0.80 and 0.95 ).

In addition, results also show (see column 4 for analyses 3 and 4 in Table 2) that the inference capabilities for the intensity measure, $P_{32}$, are very good (relative errors of less than $10 \%$ are achieved in all but one case). Furthermore, they indicate (see column 7) that the inference capabilities for the mean size of discontinuities, $\mu_{\mathrm{R}}$, is also quite good (relative errors of less than $20 \%$ are obtained in all cases), and that the estimated values of the standard deviation of the discontinuity size distribution, $\sigma_{\mathrm{R}}$, are significantly less accurate. Fortunately, however, previous research has shown (see e.g. Jimenez-Rodriguez and Sitar 2008) that $P_{32}$ and $\mu_{\mathrm{R}}$ are the parameters with the strongest influence on some important engineering problems such as the formation of removable blocks in rock slopes, hence making such inference errors on the variability of discontinuity sizes have a significantly smaller influence on results computed with the calibrated network.
Figure 8 shows an example of the inference capabilities of the GA in terms of the inferred maps of discontinuity traces. To that end, in Fig. 8 a we show the reference trace map that is assumed to be observed in a reference outcrop, and in Fig. 8b we show the trace map associated to the 'best' solution obtained with the GA. (We used a GA with fitness function defined in terms of the number of traces only, with $P_{\text {crossover }}=0.95$ and $P_{\text {mutation }}=0.03$, and with generation values given by $P_{32}=2 \mathrm{~m}^{2} / \mathrm{m}^{3}, \mu_{\mathrm{R}}=10 \mathrm{~m}$, and $\sigma_{\mathrm{R}}=4 \mathrm{~m}$; this analysis is labelled $4 \mathrm{e}$ in Table 2 , and it can be considered as a case with 'typical' accuracy, since it has estimation errors of about $5 \%$.) Results show that both trace maps are statistically very similar, and also that the number of observed and simulated traces of each type (and of each type of censored traces) are very similar as well (see captions in Fig. 8).

Figure 9 shows an example (for analysis $4 c$ ) of the evolution of the intensity parameter, $P_{32}$, corresponding to the 'best' individual (i.e., the individual with highest fitness value) in each generation. Similarly, Fig. 10 shows an example of the evolution for each generation of the mean of the discontinuity size distribution, $\mu_{R}$, for the 'best' individual in the population, whereas Fig. 11 shows the evolution of the 'best' estimation of the standard deviation of discontinuity sizes, $\sigma_{R}$. (In Figs. 9a, 10a, 11a we plot the $Y$ axis with dimensions given by the search interval $\left[x_{\min }, x_{\max }\right]$ used for parameter inference; in addition, a dashed line is employed to indicate the original parameter values employed for generation of discontinuities. 


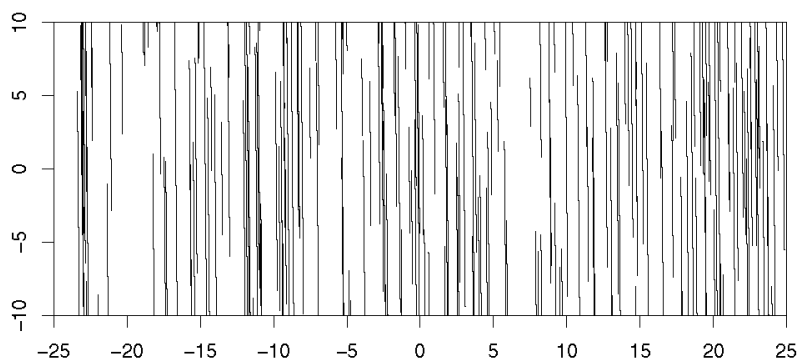

(a) Reference trace map $\left(N_{\text {total }}=215 ; N_{0}=37 ; N_{1}=164 ; N_{2}=14\right)$

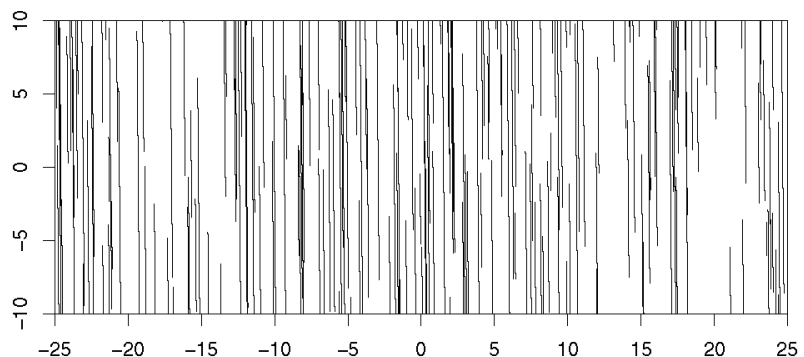

(b) Trace map simulated with the GA $\left(N_{\text {total }}=213 ; N_{0}=37 ; N_{1}=160 ; N_{2}=16\right)$

Fig. 8 Comparison between reference (observed) discontinuity trace map and the discontinuity trace map obtained as solution of the GA

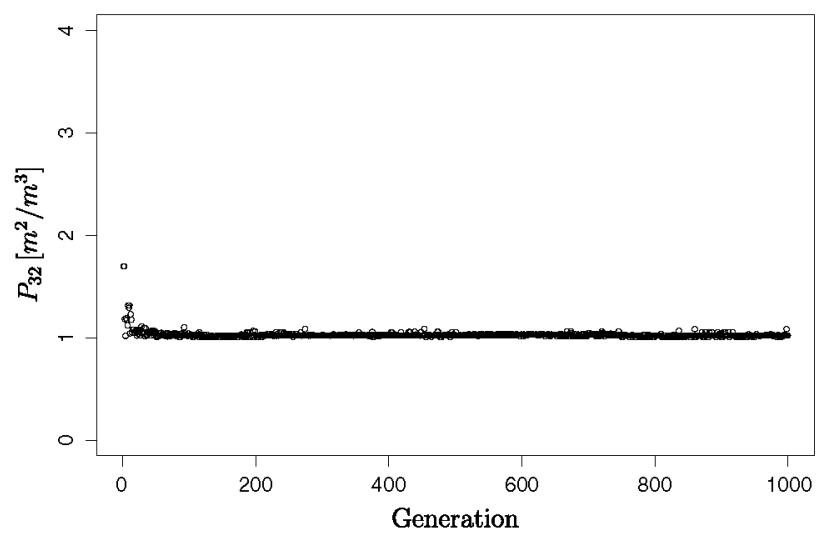

(a) Solutions within search interval

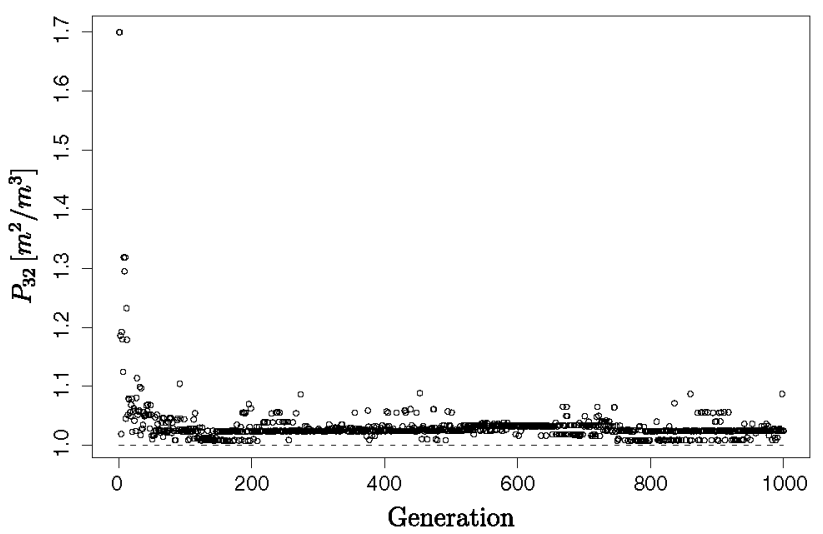

(b) Zoomed view

Fig. 9 Evolution of inferred intensity values for the best individual in successive generations of the genetic algorithm

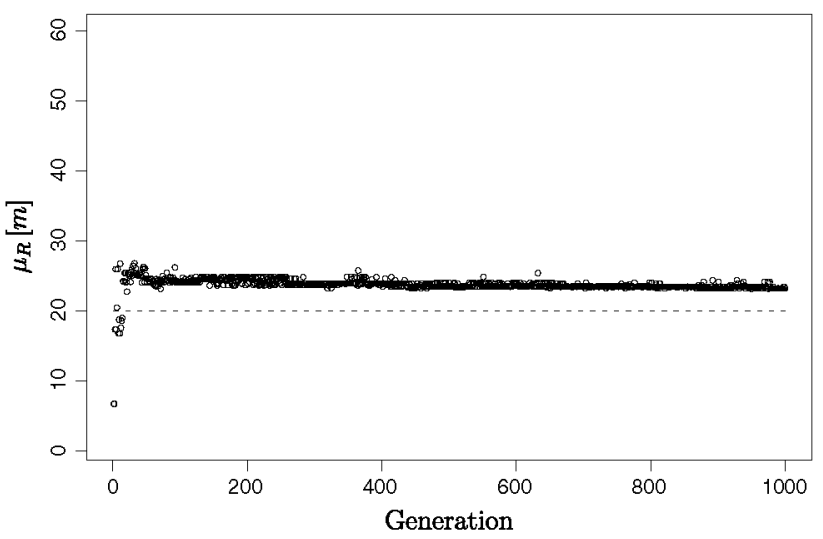

(a) Solutions within search interval

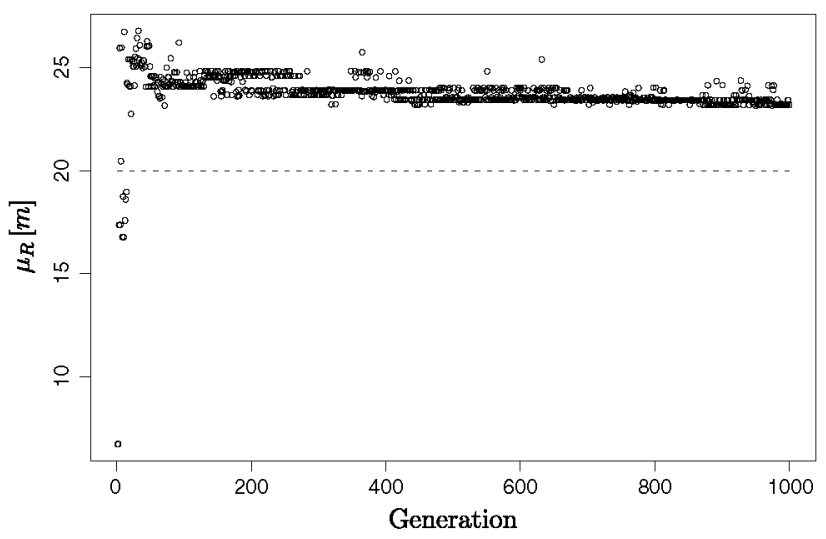

(b) Zoomed view

Fig. 10 Evolution of inferred values of the mean discontinuity size for the best individual in successive generations of the genetic algorithm

Similarly, in Figs. 9b, 10b, and 11b we plot a zoomed view of the obtained solutions for better visualization of convergence and fluctuations.)

It can be observed that, in all cases, there is an initial rapid convergence of the estimated parameter towards values very similar (within the error range discussed above and shown in Table 2) to those employed for generation of the 'observed' network used as reference in the GA. It is also interesting to note that the rate of convergence decreases after the first few generations, although it seems to continue (at a more reduced rate) even after many generations have occurred. In addition, it can be observed that, for some generations, there are (slight) oscillations of the estimated parameters values (the reason for which is discussed below). [Convergence of the algorithm with real (i.e., field) data could be expected to be somewhat slower, since the quality of field-observed trace maps would be lower and, at the same time, would be affected by aspects_-such as orientation variability within joint setsnot considered herein.] 


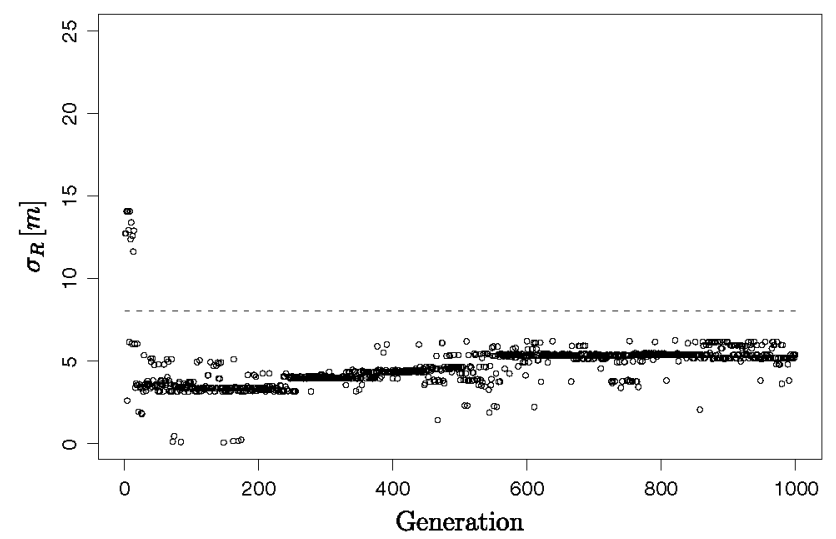

(a) Solutions within search interval

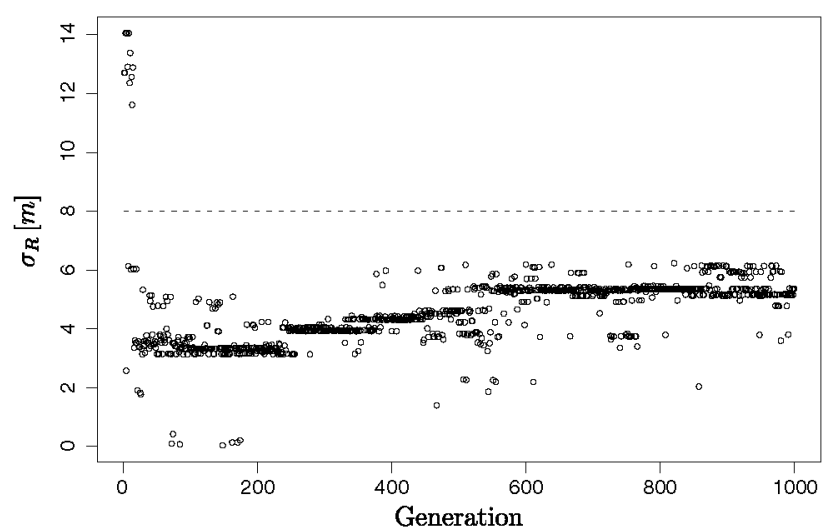

(b) Zoomed view

Fig. 11 Evolution of inferred values of the standard deviation of discontinuity sizes for the best individual in successive generations of the genetic algorithm

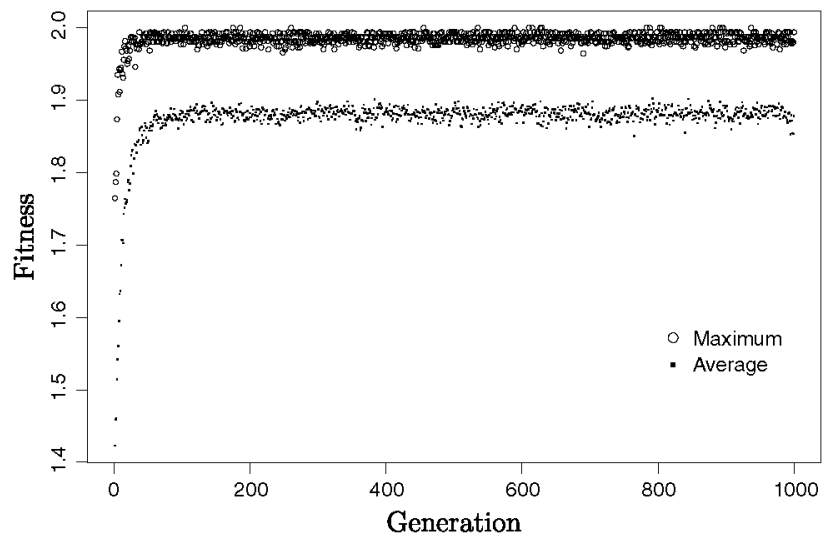

Fig. 12 Evolution of maximum (for 'best' individual) and average (for population) fitness values for each generation of the genetic algorithm

Similarly, Fig. 12 shows the evolution of the values of the fitness function for the 'best' individual in each generation. (Such individual will be therefore considered 'the solution' to the parameter estimation problem.) In addition,
Fig. 12 also shows the evolution of the average fitness for all individuals in each generation. (As expected, the average fitness is always smaller than the maximum.) As in the case of the parameter estimates, it can be observed that there is a rapid convergence in terms of the fitness of solutions during the first few generations of the algorithm, so that both the maximum and average fitness quickly reach a plateau in which small oscillations are observed.

In a general case, the slight oscillations that occur both for the estimated parameters values (Figs. 9, 10, 11) and also for the values of the fitness function (Fig. 12) could be explained as a result of the evolutionary nature of GAs unless a 'keep the best' strategy (i.e. elitism) is employed. However, in this case we are indeed keeping the solution given by the best individual of each iteration (i.e. we are using elitism) and, therefore, this is not exactly the reason for the observed oscillations. Alternatively, the explanation is that we are estimating parameters of statistical distributions using the realizations of such distributions, which are of course random. This means that different realizations of the discontinuity network performed with the same values of the statistical parameters used for generation of discontinuities will be different (because the generated discontinuities are random and, therefore, different) and, accordingly, will have different fitness values. This means that individuals (and their corresponding genotypes) have different fitness values at different moments of the generation evolution, hence explaining the observed (and slight) oscillations of the computed fitness and the estimated parameter values.

\section{Conclusions}

We present a novel approach for calibration of stochastic discontinuity network parameters based on genetic algorithms. In particular, we present an application example in which simple genetic algorithms are employed to calibrate the parameters of discontinuity networks represented with the Poisson disk model. The parameters of the model are encoded as chromosomes using a binary representation; such chromosomes evolve as successive generations of a randomly generated initial population, subjected to the operations of selection, crossover and mutation, which are defined in the genetic algorithm in a similar fashion as to how they occur in nature.

To validate the approach, we present examples of application of the method to cases in which the original parameters of the discontinuity networks are known. Using discontinuity trace maps produced by such networks, we use the proposed methodology to back-calculate the parameters of the stochastic discontinuity network, which allows us to make assessments about the inference 
capabilities of the model using different objective functions and different probabilities of crossover and mutation.

Results show that the predictive capabilities of genetic algorithms significantly depend on the type of objective function considered; in particular, results show that parameter estimates are significantly improved when the objective function only considers the total number of discontinuities observed and the number of discontinuity traces with different censoring conditions (rather than when the mean and the standard deviation of trace lengths are considered). Similarly, results suggest that the predictive capabilities of the genetic algorithm improve for relatively low values of the mutation probability $\left(P_{\text {mutation }} \approx 0.03\right.$ ), whereas changes of the crossover probability do not seem to have such a significant effect (results of similar quality being obtained for $P_{\text {crossover }}$ in the range between 0.8 and 0.95). Finally, results also show that the calibration capabilities of genetic algorithms can be acceptable for practical engineering applications, since convergence is fast and, in most cases, can be expected to provide parameter estimates with relatively small errors (of, say, less than $10-20 \%$ ) for those parameters of the network (such as intensity and the mean size of discontinuities) that have the strongest influence on many engineering applications.

\section{References}

Baecher GB, Einstein HH, Lanney NA (1977) Statistical description of rock properties and sampling. In: Wang FD, Clark GB (eds) Energy resources and excavation technology. Proceedings, 18th US symposium on rock mechanics. Colorodo School Mines Press, Golden, 5C1.1-5C1.8

Bonnet E, Bour O, Odling N, Davy P, Main I, Cowie P, Berkowitz B (2001) Scaling of fracture systems in geological media. Rev Geophys 39(3):347-383

Cai MF, Wang P, Zhao K, Zhang D (2005) Fuzzy C-means cluster analysis based on genetic algorithm for automatic identification of joint sets. Chin J Rock Mech Eng 24(3):371-376

Cravero M, Piana F, Ponti S, Tallone S, Balestro G, Morelli M (2006) Analysis of fracture patterns constrained by suitable geological model. In: Yale D, Holtz S, Breeds C, Ozbay U (eds) Proceedings of the 41st US Rock Mechanics Symposium. American Rock Mechanics Association, Golden, CO

Cui LJ, Sheng DC (2005) Genetic algorithms in probabilistic finite element analysis of geotechnical problems. Comput Geotech 32:555-563

Decker J, Mauldon M (2006) Determining size and shape of fractures from trace data using a differential evolution algorithm. In: Yale D, Holtz S, Breeds C, Ozbay U (eds) Proceedings of the 41st US Rock Mechanics Symposium. American Rock Mechanics Association, Golden, $\mathrm{CO}$

Dershowitz W, Carvalho J (1996) Key-block tunnel stability analysis using realistic fracture patterns. In: Aubertin M, Hassani F, Mitri HS (eds) Proceedings of the 2nd North American rock mechanics symposium; NARMS '96, a regional conference of ISRM; Rock mechanics tools and techniques. A.A. Balkema, Rotterdam, pp 1747-1751
Dershowitz WS, Einstein HH (1988) Characterizing rock joint geometry with joint system models. Rock Mech Rock Eng 21(1):21-51

Dershowitz WS, Herda HH (1992) Interpretation of fracture spacing and intensity. In: Tillerson JR, Wawersik WR (eds) Rock mechanics; Proceedings of the 33rd US symposium Rock mechanics. A.A. Balkema, Rotterdam, pp 757-766

Dowd PA, Martin JA, Xu C, Fowell RJ, Mardia KV (2009) A threedimensional fracture network data set for a block of granite. Int $\mathbf{J}$ Rock Mech Min Sci 46:811-818

Fahd A, Jimenez R (2008) A genetic algorithm for identification of slip surfaces with minimum reliability. In: Proceedings of the 12th international conference on computer methods and advances in geomechanics (IACMAG-2008), pp 1612-1618

Goldberg DE (1989) Genetic algorithm in search, optimization, and machine learning. Addison-Wesley, Reading

Goodman RE (1976) Methods of geological engineering in discontinuous rocks. West, St. Paul

Guan Z, Jiang Y, Tanabashi Y (2009) Rheological parameter estimation for the prediction of long-term deformations in conventional tunnelling. Tunnell Underground Space Technol 24(3):250-259

Hudson JA, Harrison JP (1997) Engineering rock mechanics: an introduction to the principles, 1st edn. Pergamon, Tarrytown

Jimenez R (2008) Fuzzy spectral clustering for identification of rock discontinuity sets. Rock Mech Rock Eng 41(6):929-939. ISSN: 0723-2632. doi:10.1007/s00603-007-0155-6

Jimenez-Rodriguez R, Sitar N (2006a) Inference of discontinuity trace length distributions using statistical graphical models. Int J Rock Mech Min Sci 43(6):877-893

Jimenez-Rodriguez R, Sitar N (2006b) A spectral method for clustering of rock discontinuity sets. Int J Rock Mech Min Sci 43(7):1052-1061. ISSN: 1365-1609. doi:10.1016/j.ijrmms.2006. 02.003

Jimenez-Rodriguez R, Sitar N (2008) Influence of stochastic discontinuity network parameters on the formation of removable blocks in rock slopes. Rock Mech Rock Eng 41(4):563-585

Kemeny J, Post R (2003) Estimating three-dimensional rock discontinuity orientation from digital images of fracture traces. Comput Geosci 29:65-77

Kulatilake P, Wathugala DN, Stephansson O (1993) Joint network modelling with a validation exercise in Stripa Mine, Sweden. Int J Rock Mech Mining Sci Geomech Abstr 30(5):503-526

Kulatilake P, Wu TH (1986) Relation between discontinuity size and trace length. In: Hartman HH (ed) Rock mechanics; proceedings of the 27th US symposium. A.A. Balkema, Rotterdam, pp 130-133

Kulatilake PHSW, Um J-G, Wang M, Escandon RF, Narvaiz J (2003) Stochastic fracture geometry modeling in 3-D including validations for a part of Arrowhead east tunnel, California, USA. Eng Geol 70(1-2):131-155

Kuszmaul JS (1999) Estimating keyblock sizes in underground excavations: accounting for joint set spacing. Int $\mathbf{J}$ Rock Mech Min Sci 36(2):217-232

La Pointe PR (1993) Pattern analysis and simulation of joints for rock engineering. In: Hudson JA (ed) Comprehensive rock engineering, rock testing and site characterization, vol 3. Pergamon, Oxford, pp 215-239

La Pointe PR, Wallmann PC, Dershowitz WS (1993) Stochastic estimation of fracture size through simulated sampling. Int J Rock Mech Min Sci Geomech Abstr 30(7):1611-1617

Lee JS, Veneziano D, Einstein HH (1990) Hierarchical fracture trace model. In: Hustrulid WA, Johnson GA (eds) Rock mechanics; contributions and challenges; proceedings of the 31st US symposium, A.A. Balkema, Rotterdam, pp 261-268

Levasseur S, Malecot Y, Boulon M, Flavigny E (2008) Soil parameter identification using a genetic algorithm. Int $J$ Numer Anal Methods Geomech 32(2):189-213 
Levasseur S, Malecot Y, Boulon M, Flavigny E (2010) Statistical inverse analysis based on genetic algorithm and principal component analysis: applications to excavation problems and pressuremeter tests. Int J Numer Anal Methods Geomech 34:471-491

Lyman GJ (2003a) Rock fracture mean trace length estimation and confidence interval calculation using maximum likelihood methods. Int J Rock Mech Min Sci 40:825-832

Lyman GJ (2003b) Stereological and other methods applied to rock joint size estimation-does crofton's theorem apply? Math Geol $35(1): 9-23$

Mauldon M (1998) Estimating mean fracture trace length and density from observations in convex windows. Rock Mech Rock Eng 31(4):201-216

Meyer T, Einstein HH (2002) Geologic stochastic modeling and connectivity assessment of fracture systems in the Boston area. Rock Mech Rock Eng 35(1):23-44

Mitchell M. (1996) An introduction to genetic algorithms. MIT Press, Cambridge

Munier R (2004) Statistical analysis of fracture data, adapted for modelling discrete fracture networks-version 2. Technical Report, SKB Rapport R-04-66; ISSN 1402-3091, The Swedish Nuclear Fuel and Waste Management Company (SKB), Stockholm, Sweden. http://www.skb.se/upload/publications/pdf/R-0466webb.pdf. Accessed 03 Aug 2011

Pahl PJ (1981) Estimating the mean length of discontinuity traces. Int J Rock Mech Min Sci Geomech Abstr 18(3):221-228

Park H, West TR (2001) Development of a probabilistic approach for rock wedge stability. Eng Geol 59:233-251

Park H-J, West TR, Woo I (2005) Probabilistic analysis of rock slope stability and random properties of discontinuity parameters, Interstate Highway 40, Western North Carolina, USA. Eng Geol $79(3-4): 230-250$

Priest SD (2004) Determination of discontinuity size distributions from scanline data. Rock Mech Rock Eng 37(5):347-368
Simpson AR, Priest SD (1993) The application of genetic algorithms to optimisation problems in geotechnics. Comput Geotech 15(1):1-19

Song JJ, Lee CI, Seto M (2001) Stability analysis of rock blocks around a tunnel using a statistical joint modeling technique. Tunnell Undergr Space Technol 16(4):341-351

Starzec P, Andersson J (2002) Probabilistic predictions regarding key blocks using stochastic discrete fracture networks: example from a rock cavern in south-east Sweden. Bull Eng Geol Environ 61(4):363-378

Tokhmechi B, Memarian H, Moshiri BB, Rasouli V, Noubari H (2011) Investigating the validity of conventional joint set clustering methods. Eng Geol 118(3-4):75-81

Tonon F, Chen S (2007) Closed-form and numerical solutions for the probability distribution function of fracture diameters. Int J Rock Mech Min Sci 44:332-350

Villaescusa E, Brown ET (1992) Maximum likelihood estimation of joint size from trace length measurements. Rock Mech Rock Eng 25(2):67-87

Xue J-F, Gavin K (2007) Simultaneous determination of critical slip surface and reliability index for slopes. $\mathbf{J}$ Geotech Geoenviron Eng (ASCE), 133(7):878-886

Zhang L, Einstein HH (1998) Estimating the mean trace length of rock discontinuities. Rock Mech Rock Eng 31(4):217-235

Zhang L, Einstein HH, Dershowitz WS (2002) Stereological relationship between trace length and size distribution of elliptical discontinuities. Géotechnique 52(6):419-433

Zhang LY, Einstein HH (2000) Estimating the intensity of rock discontinuities. Int J Rock Mech Min Sci 37(5):819-837

Zolfaghari AR, Heath AC, McCombie PF (2005) Simple genetic algorithm search for critical non-circular failure surface in slope stability analysis. Comput Geotechn 32:139-152 